\title{
Relación entre rasgos de personalidad y valores personales en pacientes dependientes de la cocaína
}

\section{Relation between personality traits and personal values in cocaine-dependent patients}

\author{
| Jesús SaIz*; José Luis Álvaro*; Isabel Martínez ${ }^{* *}$
}

$\left.\right|^{*} \quad \begin{aligned} & \text { Universidad Complutense de Madrid. } \\ & \text { ** Universidad de Castilla-La Mancha. }\end{aligned}$

Enviar correspondencia a:

Jesús Saiz. D. 2306B, Departamento de Psicología Social.

Facultad de Psicología. Campus de Somosaguas.

Universidad Complutense de Madrid. 28223 Madrid.

E-mail: jesus.saiz@psi.ucm.es

\section{RESUMEN}

Objetivo: Describir la relación que guardan los valores personales y los rasgos de personalidad en pacientes consumidores de cocaína y conocer su especificidad en la explicación de diferentes constructos.

Método: Se realizó un estudio en el que se exploró la relación entre estas variables en un grupo de 230 participantes que se encontraban en tratamiento por dependencia a la cocaína, utilizando el Portrait Values Questionnaire para medir los valores personales y el Big-Five Factors Questionnaire para medir los rasgos de personalidad. Además, se examinó la relación de rasgos y valores con las variables "grado de satisfacción con la vida" (satisfacción vital) y "pertenencia a alguna asociación religiosa" (religiosidad).

Resultados: Se encontró una asociación significativa entre valores personales y rasgos de personalidad, a la vez que se distinguieron sus diferencias conceptuales y empíricas, al hallar que los valores personales explican mejor la pertenencia a alguna asociación religiosa, mientras que los rasgos de personalidad explican mejor el grado de satisfacción con la vida. Por tanto, se comprueba que los valores personales explican mejor los comportamientos que dependen de mayor control cognitivo, mientras que los rasgos de personalidad afectarian más a tendencias y conductas sujetas a un reducido control cognitivo.

Conclusiones: Tomando en consideración la relación entre ambos constructos y dado que el consumo de cocaína se asocia tanto con alto como con bajo control cognitivo, resultará apropiado tener en cuenta para la comprensión del consumo de cocaína, la aportación explicativa de los valores personales y de los rasgos de personalidad de forma complementaria.

Palabras clave: Valores personales, rasgos de personalidad, consumo de cocaína, religiosidad, satisfacción vital. recibido: mayo 2010 aceptado: octubre 2010

\section{ABSTRACT}

Objective: To describe the relationship between personal values and personality traits in cocaine-using patients and analyze their specificity in the explanation of different types of constructs.

Method: A study was carried out to explore the association between these variables in a group of 230 patients receiving treatment for cocaine dependence. The Portrait Values Questionnaire was used for measuring personal values, while the Big-Five Factors Questionnaire was used to measure personality traits. In addition, we explored the relationship of values and traits with the variables "degree of satisfaction with life" (life satisfaction) and "belonging to a religious association" (religiosity).

Results: A significant association was found between personal values and personality traits. At the same time, their conceptual and empirical differences were revealed, as it was demonstrated that personal values better explain "belonging to a religious association", whilst personality traits better explain "degree of satisfaction with life". Thus, it was found that personal values better explain behaviours that depend on greater cognitive control, while personality traits would have more influence on tendencies and behaviours that are subject to lower cognitive control levels.

Conclusions: Considering the relationship between the two constructs, and given that cocaine use is associated with both high and low cognitive control, in explanations of cocaine use it would be appropriate to take into account the explanatory contribution of personal values and personality traits in a complementary way.

Key words: Personal values, personality traits, cocaine use, religiosity, life satisfaction. 


\section{INTRODUCCIÓN}

$\mathrm{E}$ consumo de cocaína en España y el número de personas tratadas por abuso o dependencia de esta sustancia se ha incrementado notablemente en los últimos años, tal y como se detalla en el último informe del Observatorio Español sobre Drogas [OED] (2007). Esto acrecienta la necesidad de contar con instrumentos de evaluación, tratamiento y prevención, basados en la evidencia científica, que faciliten intervenciones más eficientes. Según las recomendaciones de algunos manuales y estudios (Becoña, 2002; Galdós, 2009; Morales, Ariza, Nebot, Pérez y Sánchez, 2008; Saiz, 2007) una estrategia conveniente para una intervención preventiva eficaz es la consideración del mayor número de posibles factores relacionados.

Dentro de los factores psicosociales que en la bibliografía científica han mostrado tener especial relevancia en la descripción y explicación del consumo de sustancias se encuentran los rasgos de personalidad (Pedrero, 2002; Terracciano, Löckenhoff, Crum, Bienvenu y Costa, 2008) y los valores personales (Berjano y García, 1996; Galdós y Sánchez, 2010; Luciano, Páez-Blarrina y Valdivia-Salas, 2010; Pérez, Mota, y Arroyo, 2008; Pons y Berjano, 1999; Liu, Yu, Cottrell, Lunn, Deveaux, Brathwaite, et al., 2007). No obstante, los trabajos que relacionan estas variables con el comportamiento de adicción a la cocaína no analizan la interacción de estas dos variables en consumidores de sustancias. Por otra parte, las investigaciones que comparan ambas variables han sido realizadas en muestras universitarias, no clínicas, y provenientes de culturas diferentes a la española (Bilsky y Schwartz, 1994; Caprara, Vecchione y Schwartz, 2009; Luk y Bond, 1993; Olver y Mooradian, 2003; Roccas, Sagiv, Schwartz y Knafo, 2002).

En el presente estudio se plantea describir la relación que guardan los valores personales y los rasgos de personalidad como variables complementarias en un grupo de personas consumidoras de cocaína en tratamiento, a la vez que se tiene en cuenta la especificidad conceptual y empírica de ambas variables como predictoras de comportamientos diferentes. Los valores de las personas consumidoras de cocaína se analizaron siguiendo la Teoría de los Valores Personales de Schwartz (2001), mientras que los rasgos de personalidad se examinaron partiendo del Modelo de los Cinco Factores de Personalidad (McCrae y John, 1992; Tupes y Cristal, 1961).

\section{Teoría de los Valores Personales de Schwartz}

Una de las teorías de los valores personales que cuenta con mayor apoyo empírico y solidez teórica en la actualidad es la elaborada por Schwartz y Bilsky (1987) y revisada posteriormente por Schwartz (2001). En esta teoría, los valores son definidos como metas o fines deseables que son generalizables a distintas situaciones, se organizan de forma jerárquica y guían la conducta de las personas, pudiéndose distinguir diez tipos motivacionales de valores: 1) Poder, definido como estatus social, control sobre las personas y recursos; 2) Logro, referido al éxito personal mediante la demostración de competencia según criterios sociales; 3) Hedonismo, definido como placer y gratificación sensual para uno mismo; 4) Estimulación, definido como entusiasmo, novedad y reto en la vida; 5) Autodirección, referido al pensamiento independiente y a la elección de la acción, creatividad y exploración; 6) Universalismo, que se define como comprensión, aprecio, tolerancia y protección del bienestar de todas las personas y de la naturaleza; 7) Benevolencia, definido como preservación e intensificación del bienestar de todas las personas con las que uno está en contacto personal frecuente; 8) Tradición, definido como respeto, compromiso y aceptación de las costumbres e ideas que proporcionan la cultura tradicional o la religión; 9) Conformidad, referido a la restricción de las acciones, inclinaciones e impulsos que pudiesen molestar o herir a otros y violar expectativas o normas sociales; y 10) Seguridad, que se define como armonía, estabilidad y seguridad en la sociedad, de las relaciones y para sí mismo.

El modelo de Schwartz (2001) establece la existencia de una serie de relaciones dinámicas entre estos valores, considerando que algunos son compatibles entre ellos, mientras que otros son incompatibles. De esta manera, se consideran dos dimensiones bipolares básicas: la primera dimensión se define por Apertura al cambio (que incluye los valores de autodirección y estimulación) versus Conservación (que incluye los valores de tradición, conformidad y seguridad) y agrupa los valores en función de si comparten una cierta inclinación al cambio o de si se interesan por conservar el status quo; mientras que la segunda dimensión opone Autopromoción (que incluye poder y logro) a Autotrascendencia (que comprende los valores de universalismo y benevolencia), agrupando aquellos valores que benefician al individuo frente a los que benefician sobre todo a la colectividad. El valor hedonismo está relacionado tanto con apertura al cambio como con autopromoción (figura 1).

La relación de los valores del modelo de Schwartz con el consumo de sustancias ha sido puesta de manifiesto en diferentes estudios. Por ejemplo, Liu et al. (2007) encontraron que los varones con puntuaciones altas en valores de

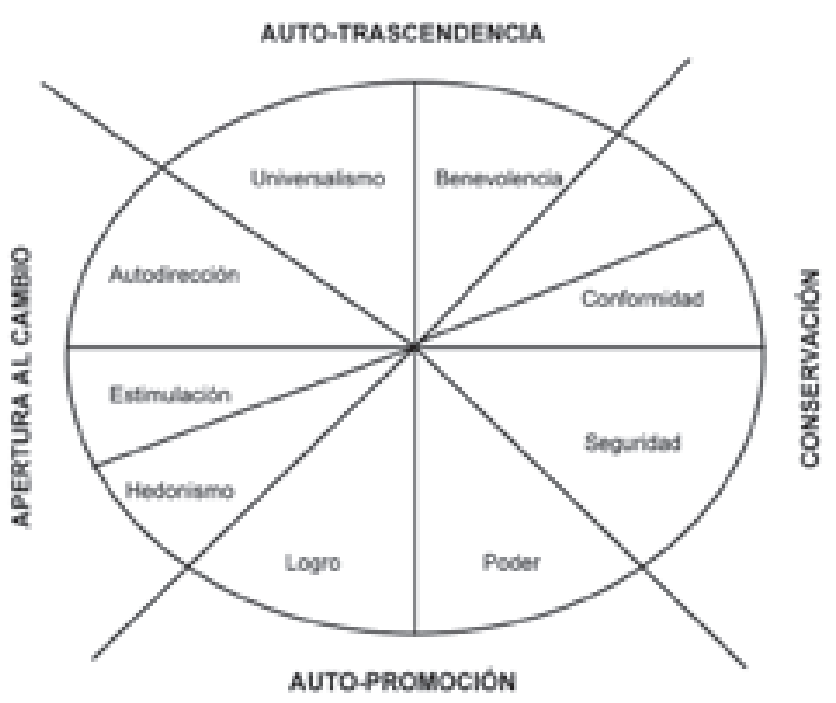

Figura 1. Modelo teórico de las relaciones entre los valores personales agrupados en dimensiones bipolares en función de su similitud (Schwartz, 2001). 
autopromoción y las mujeres con puntuaciones altas en valores de apertura al cambio y bajas en valores de conservación, estaban más involucrados en comportamientos como el consumo abusivo de alcohol y drogas. Por su parte, Pons y Berjano (1999) también describieron que el consumo abusivo de alcohol estaba mediado por el mantenimiento de los valores de hedonismo, estimulación, tradición y conformidad y alejado del mantenimiento de los valores de benevolencia y universalismo. Igualmente en el estudio de Galdós y Sánchez (2010), se comprobó que las personas que se encontraban bajo tratamiento para abandonar la adicción a la cocaína, tendian a mostrar puntuaciones significativamente menores en los valores de apertura al cambio y mayores en los valores de conservación que las personas consumidoras de cocaína que no estaban en tratamiento para abandonar su adicción.

\section{Modelo de los Cinco Factores de Personalidad}

El Modelo de los Cinco Factores de Personalidad (Digman y Takemoto, 1981; Digman, 1990; Goldberg, 1990; McCrae y John, 1992; Tupes y Cristal, 1961) es un eficaz modelo descriptivo de la personalidad, que posee tres características que lo distinguen: "...integra una amplia variedad de constructos de personalidad, facilitando la comunicación entre investigadores de diferentes orientaciones; es comprensivo, dando una base para la exploración sistemática de las relaciones entre personalidad y otros fenómenos; y es eficiente, proveyendo una descripción global de la personalidad con tan sólo cinco puntuaciones" (McCrae y John, 1992, p. 206).

Según el modelo de los cinco factores cualquier tipo de personalidad puede ser explicada a partir de cinco amplias dimensiones: 1) Extraversión o Energía: una persona extrovertida es sociable, amante de la diversión, dinámica, activa, energética, amistosa, dominante y locuaz; 2) Afabilidad o Amabilidad: envuelve aspectos como el altruismo, el cuidado emocional, los celos, la indiferencia o la sensibilidad hacia los demás; 3) Tesón, o responsabilidad, considerado como la voluntad de lograr algo e incluye reflexión, escrupulosidad, orden y diligencia; 4) Estabilidad Emocional o Neuroticismo: individuos altos en estabilidad emocional (o bajos en neuroticismo) son tranquilos, fuertes, fríos y emocionalmente controlados; y 5) Apertura Mental o Intelecto, caracterizado por la originalidad, la imaginación, la personalidad culta, informada y con interés por cosas nuevas y culturas diferentes.

La relación entre los rasgos de personalidad y el consumo de sustancias también ha sido explorada en distintos estudios. Así, Berrocal, Ortiz-Tallo, Fierro y Jiménez (2001) hallaron en un grupo de personas dependientes de la heroína, que éstos tendían a puntuar más bajo en los rasgos de personalidad tesón y apertura mental, medidos con el Big-Five Factors Questionnaire. Utilizando esta misma medida, Pedrero (2002) encontró una tendencia a mostrar puntuaciones bajas en estabilidad emocional en personas demandantes de tratamiento por abuso o dependencia de alcohol, cocaína o heroína. Del mismo modo, Terracciano, Löckenhoff, Crum, Bienvenu y Costa (2008) hallaron puntuaciones especialmente bajas en neuroticismo o estabilidad emocional y tesón o responsabilidad en consumidores y ex-consumidores de cocaína y/o heroína.

Tanto los valores personales como los rasgos de personalidad pueden ser considerados como características básicas de la identidad individual que, en condiciones normales, no se espera sufran importantes modificaciones a lo largo de la vida (McCrae, 2001; Schwartz, 2001). No obstante, como han señalado Bilsky y Schwartz (1994), las principales diferencias entre ambos conceptos residen en que: 1) los rasgos de personalidad son típicamente vistos como patrones de conducta observables, mientras que los valores son criterios que los individuos usan para juzgar la deseabilidad de la conducta, la gente y los acontecimientos; 2) los rasgos de personalidad varían en función de la intensidad o grado en que son mostrados por los individuos, mientras que los valores varían en función de la importancia que los individuos atribuyen a objetivos particulares; $y, 3$ ) los rasgos de personalidad describen acciones que presumiblemente surgen de "la forma en como la gente es" sin importar sus intenciones, mientras que los valores se refieren a los objetivos intencionales de los individuos. Por lo tanto, mientras que los rasgos de personalidad son atribuciones que se realizan a las personas según su comportamiento observable, los valores son experimentados como lo que una persona juzga o considera importante.

Sin embargo, varios estudios han puesto de manifiesto que estos dos constructos pueden estar relacionados. Algunas investigaciones llevadas a cabo con estudiantes universitarios en Estados Unidos e Israel muestran que, en general, la extroversión tiende a correlacionar con logro, aunque también hay estudios que la relacionan con el hedonismo y la estimulación (Bilsky y Schwartz, 1994); la apertura a la experiencia con autodirección y universalismo; la afabilidad positivamente con benevolencia y negativamente con poder; el tesón se relaciona con logro y seguridad; mientras que la estabilidad emocional tiende a mostrar una baja asociación con el conjunto de los valores de Schwartz (Roccas et al., 2002; Olver y Mooradian, 2003). Las relaciones entre ambas variables nunca han sido exploradas ni en la población española ni en una población clínica, como son los consumidores de cocaína, en donde la relación entre estos dos constructos podría tener especial relevancia. Dado que ambas variables han mostrado estar relacionadas tanto con el consumo de sustancias como con el abandono de este consumo (Galdós y Sánchez, 2010; Pedrero 2002; Pons, Berjano y Garcia, 1996) su análisis conjunto podría proporcionar un perfil más completo de los factores psicosociales que caracterizan al consumidor de cocaína.

A partir de las diferentes dimensiones de los valores de Schwartz y los rasgos de personalidad contemplados en el modelo de los cinco factores, y teniendo en cuenta los resultados obtenidos con poblaciones no clínicas, se han formulado las siguientes hipótesis respecto a la relación entre ambos constructos en personas consumidoras de cocaína:

Ho1. Dado que el rasgo energía implica un comportamiento estrechamente orientado hacia la acción 
inmediata se espera una asociación positiva con los valores de la dimensión autopromoción (logro, poder y hedonismo).

Ho2. Puesto que el rasgo apertura mental sugiere un conjunto de comportamientos de aceptación de distintos estilos de vida, es previsible que muestre una correlación positiva con los valores de la dimensión de apertura al cambio (autodirección, estimulación y hedonismo) y autotrascendencia (universalismo y benevolencia).

Ho3. Teniendo en cuenta que el valor afabilidad se refiere a comportamientos favorables al endogrupo, se prevé que muestre una correlación positiva con los valores de autotrascendencia (universalismo y benevolencia).

Ho4. Considerando que el rasgo tesón sugiere acciones persistentes y continuadas en el tiempo, se espera que muestre una correlación positiva con los valores de la dimensión autopromoción (logro, poder y hedonismo).

Ho5. Dado que el rasgo estabilidad emocional pone el énfasis en una situación personal libre de amenazas, se espera que guarde una relación positiva con los valores de la dimensión conservación (tradición, conformidad y seguridad).

Por otra parte, a pesar de que esperamos que ambos constructos estén correlacionados, los valores personales y los rasgos de personalidad son variables diferentes $y$, como tales, pueden jugar distintos papeles en la prevención y el tratamiento por dependencia de la cocaína. Con el fin de contrastar las diferencias conceptuales y empíricas de ambos constructos se estudió su relación con otras dos variables (religiosidad y satisfacción vital) que ya han sido utilizadas anteriormente por Roccas et al. (2002) con un objetivo similar. Respecto a la relación de estas variables con los valores personales y los rasgos de personalidad, se plantean las siguientes hipótesis:

Ho6. Dado que "el grado de satisfacción con la vida" es una variable compleja que comprende mayoritariamente elementos que escapan a la acción voluntaria de la persona, esperamos que exista una mayor relación entre los rasgos de personalidad y esta variable.

Ho7. En cambio, teniendo en cuenta que "la pertenencia a alguna asociación religiosa" podría ser considerada como una acción razonada y voluntaria de la persona, se espera que los valores personales correlacionen de una manera más importante con esta otra variable.

\section{MÉTODO}

\section{Muestra}

La muestra estuvo compuesta por 233 participantes dependientes de la cocaína, de 15 a 39 años, siendo la mayoría varones (82,3\%). Este grupo está formado por personas que acudian a distintos Centros de Atención Integral a
Drogodependientes (CAID) y a otros centros de rehabilitación de la Comunidad de Madrid (públicos y privados)'.

\section{Procedimiento}

La aplicación de los instrumentos de medida se realizó de manera colectiva en los centros de tratamiento a los que acudian los participantes, en aproximadamente la mitad de la muestra. Sin embargo, en las ocasiones en las que no fue posible reunir a los grupos, fueron profesionales entrenados los encargados de administrar los cuestionarios en estos mismos centros de tratamiento. Todos los participantes fueron invitados a participar voluntariamente en el estudio por su profesional de referencia, asegurándoles la confidencialidad y anonimato por escrito.

\section{Instrumentos}

Para medir los valores personales de los participantes en el estudio se utilizó el Portrait Values Questionnaire (PVO), desarrollado por Schwartz, Melech, Lehmann, Burgess, Harris y Owens (2001) y basado en el modelo teórico de Schwartz (2001). Este instrumento representa una simplificación del cuestionario para la medición de los valores Schwartz Value Survey (SVS) (Schwartz, 2001), desarrollado con una muestra total de 25.863 personas procedentes de 44 países y que ha mostrado una alta validez transcultural (Martínez y García, 2007; Martínez y García, 2008; Schwartz, Verkasalo, Antonovsky y Sagiv, 1997; Spini, 2003). La contestación del PVO resulta más sencilla $y$, además, reduce el número de ítems de 56 a 29, teniendo un tiempo de respuesta estimado de 10 minutos.

Utilizando entre 2 y 5 ítems para cada factor, este cuestionario mide los valores de conformidad (e. g., "Es importante ser educado/a con los demás siempre"), autodirección (e. g., "Imaginar nuevas ideas y ser creativo es importante. Le gusta ser original al hacer las cosas"), logro (e. g., "Conseguir mucho éxito es importante. Le gusta destacar e impresionar a los demás"), tradición (e. g., "Piensa que es importante hacer las cosas de la forma en que las ha aprendido de su familia. Quiere seguir sus costumbres y tradiciones"), universalismo (e. g., "Cree que es importante que todas las personas del mundo deban de ser tratadas por igual. Quiere justicia para todos/as, incluso para las personas a las que no conoce"), estimulación (e. g., "Busca aventuras y le gusta arriesgarse. Quiere tener una vida llena de novedades"), seguridad (e. g., "La seguridad de su país es muy importante. Quiere que su país esté a salvo de sus enemigos"), benevolencia (e. g., "Siempre quiere ayudar a las personas cercanas. Es muy importante cuidar de las personas a las que conoce y quiere"), poder (e. g., "Le gusta estar a cargo de otras personas y decirles lo que tienen que hacer. Es decir, quiere que la gente haga lo que dice") y hedonismo (e. g., "Quiere disfrutar realmente de la vida. Divertirse es muy importante").

1 CAIC del Hospital San Juan de Dios, CAID de Alcalá de Henares, CAID de Majadahonda, CAID de Móstoles, CAID de Usera, Centro de Tratamiento El Olivar, Comunidad Terapéutica Dianova, Comunidad Terapéutica Narconón, Federación de Alcohólicos de la Comunidad de Madrid, Narcóticos Anónimos y Proyecto Hombre. 
EI PVQ hace uso de la evaluación escalar, siendo el rango de 1 (no se parece nada a mí) a 6 (se parece mucho a mí). La versión española del PVQ ha sido validada por Saiz (2008) y Zlobina (2004) y puede consultarse, también, en el trabajo de Gómez, Gaviria y Fernández (2006). El coeficiente de fiabilidad de la prueba fue de 0,81, mientras que el coeficiente de fiabilidad para cada dimensión de valor fue: conformidad 0,45 ; autodirección 0,44 ; logro 0,69; tradición 0,41 ; universalismo 0,63 ; estimulación 0,64 ; seguridad 0,42 ; benevolencia 0,56; poder 0,43 y hedonismo 0,62. Estos indices de fiabilidad son similares a los observados para estos tipos de valor en muestras similares (Martínez y García, 2007; Roccas et al., 2002).

Para medir los rasgos de personalidad se aplicó el Big-Five Factors Questionnaire (BFQ) (Caprara, Barbaranelli y Borgogni, 2001). Esta medida facilita un perfil de los rasgos o factores de personalidad. Basándose en el Modelo de los Cinco Factores de Personalidad (Digman, 1990; Goldberg, 1990; McCrae y John, 1992), otorga puntuaciones para estos cinco factores de personalidad y diez subfactores. El BFO emplea una escala de respuesta tipo Likert del 1 al 5, en donde el 1 significa "completamente falso para mí" y el 5 "completamente verdadero para mí". A través de 120 ítems (24 para cada factor), el BFO mide los rasgos de energía (e. g., "A menudo estoy completamente absorbido por mis compromisos y actividades"), afabilidad (e. g., "Creo que todas las personas tienen algo de bueno"); tesón (e. g., "Si fracaso en algo, lo intento de nuevo hasta conseguirlo"); estabilidad emocional (e. g., "Tiendo a implicarme demasiado cuando alguien me cuenta sus problemas") y apertura mental (e. g., "Estoy siempre informado sobre lo que sucede en el mundo").

La versión española del cuestionario ha sido validada por Bermúdez (en Caprara Caprara, Barbaranelli y Borgogni, 2001) con una muestra de 2000 personas. La validación de esta escala con personas dependientes de sustancias ha sido llevada a cabo por Pedrero (2003). El coeficiente de fiabilidad de la prueba es de 0,82 , mientras que los coeficientes para cada factor fueron: energía 0,66; afabilidad 0,53; tesón 0,56; estabilidad emocional 0,80 y apertura mental 0,56.

El grado de satisfacción con la vida se evaluó con un ítem que planteaba al participante directamente: "¿Cuál sería tu grado de satisfacción con tu vida presente?", teniendo como opciones de respuesta "Nada satisfecho, Poco satisfecho, Satisfecho, Bastante satisfecho y Muy satisfecho". La pertenencia a alguna asociación religiosa se evaluó también de forma directa con un ítem que planteaba: "¿Perteneces a alguna iglesia, parroquia u otra asociación religiosa?", en donde las opciones de respuesta eran: "Si; No, pero soy creyente; y No".

\section{RESULTADOS}

\section{Relaciones entre rasgos de personalidad y valores personales}

Tal y como se expone en la tabla 1, donde se muestran las correlaciones significativas entre los cinco factores de personalidad y los diez valores analizados en el estudio, los resultados obtenidos confirman que mayoritariamente se cumplen las hipótesis planteadas sobre la relación entre estos dos constructos, además de otras relaciones que se detallan en esta tabla. Como puede observarse, el rasgo energía correlacionó positivamente con los valores de la dimensión autopromoción, logro $(r=0,42, p<0,01)$ y poder $(r=0,24, p<0,01)$. Además, correlacionó con los valores de autodirección $(r=0,28, p<0,01)$, estimulación $(r=0,25, p<$ $0,01)$, benevolencia $(r=0,18, p<0,01)$ y tradición $(r=-0,12, p$ $<0,05)$. El rasgo apertura mental correlacionó positivamente con los valores de apertura al cambio, autodirección ( $r=$ $0,35, p<0,01)$ y estimulación $(r=0,27, p<0,01)$ y con los valores de la dimensión de autotrascendencia, universalismo $(r=0,21, p<0,01)$ y benevolencia $(r=0,13, p<0,05)$. El rasgo afabilidad correlacionó positivamente con el valor de la dimensión de autopromoción, benevolencia $(r=0,25, p<$ $0,01)$. Además, también correlacionó con universalismo $(r=$ $0,38, p<0,01)$, seguridad $(r=0,16, p<0,01)$, conformidad $(r$ $=0,15, p<0,05)$ y autodirección $(r=0,15, p<0,05)$. El rasgo tesón no mostró ninguna correlación con los valores de la dimensión de autopromoción. Sin embargo, sí correlacionó positivamente con valores de las tres restantes dimensiones: seguridad $(r=0,29, p<0,01)$, autodirección $(r=0,32, p<$ $0,01)$, benevolencia $(r=0,27, p<0,01)$ y universalismo $(r=$ $0,20, p<0,01)$. Finalmente, tal y como se preveía, el rasgo estabilidad emocional mostró una correlación significativa con el valor de la dimensión conservación, seguridad ( $r=$ $0,14, p<0,05)$.

\section{Relaciones de rasgos de personalidad y valores personales con satisfacción vital y religiosidad}

Como puede observarse en la tabla 1, los rasgos de personalidad correlacionaron de forma más importante con "el grado de satisfacción con la vida". Tres de los cinco rasgos de personalidad analizados, tesón $(r=0,37, p<0,01)$, estabilidad emocional $(r=0,26, p<0,01)$ y energía $(r=0,17, p$ $<0,01)$, correlacionaron significativamente con esta variable. Los valores que también correlacionaron con "el grado de satisfacción con la vida" fueron: benevolencia $(r=0,24, p<$ $0,01)$, tradición $(r=0,22, p<0,01)$, conformidad $(r=0,18$, $p<0,01)$, seguridad $(r=0,14, p<0,01)$ y autodirección $(r=$ $0,13, p<0,05)$. Por otra parte, ningún rasgo de personalidad correlacionó con la "pertenencia a una asociación religiosa", mientras que sí lo hicieron los valores personales tradición $(r$ $=0,22, p<0,01)$ y poder $(r=0,15, p<0,05)$.

Adicionalmente, se realizaron dos análisis de regresión lineal con el fin de determinar qué variable (rasgos de personalidad o valores personales) explicaba mejor el "grado de satisfacción con la vida" y la "pertenencia a alguna asociación religiosa". Los 5 rasgos de personalidad del instrumento BFO y los 10 valores personales del PVQ se utilizaron (en diferentes análisis) como variables independientes. Tal y como se observa en la tabla 2, los rasgos de personalidad explicaron un mayor porcentaje de la varianza que los valores (16\% versus $8 \%$ ) en el caso del "grado de satisfacción con la vida", mientras que en el caso de la "pertenencia a alguna asociación religiosa", 
Tabla 1.

Correlaciones significativas entre valores y rasgos de personalidad con satisfacción con la vida y con pertenencia a alguna asociación religiosa $(N=230)$

\begin{tabular}{|c|c|c|c|c|c|c|c|}
\hline & Energía & Apertura Mental & Afabilidad & Tesón & Estabilidad Emocional & $\begin{array}{l}\text { Satisfacción con } \\
\text { la vida }\end{array}$ & Asociación Religiosa \\
\hline Conformidad & & & $0,15^{(*)}$ & & & $0,18^{(+*)}$ & \\
\hline Autodirección & $0,28^{(-)}$ & $0,35^{(*)}$ & $0,15^{(*)}$ & $0,32^{(-)}$ & & $0,13^{(*)}$ & \\
\hline Logro & $0,42^{(-)}$ & & & & & & \\
\hline Tradición & $0,12^{(+)}$ & & & & & $0,22^{(+)}$ & $0,22^{(+)}$ \\
\hline Universalismo & & $0,21^{(*)}$ & $0,38^{(*)}$ & $0,20^{(-)}$ & & & \\
\hline Estimulación & $0,25^{(+)}$ & $0,27^{(-)}$ & & & & & \\
\hline Seguridad & & & $0,16^{(+)}$ & $0,29^{(-)}$ & $0,14^{(*)}$ & $0,14^{(*)}$ & \\
\hline Benevolencia & $0,18^{(*)}$ & $0,13^{(*)}$ & $0,25^{(*)}$ & $0,27^{(*)}$ & & $0,24^{(*)}$ & \\
\hline Poder & $0,24^{(*)}$ & & & & & & $0,15^{(*)}$ \\
\hline \multicolumn{8}{|l|}{ Hedonismo } \\
\hline Satisfacción con la vida & $0,17^{(*)}$ & & & $0,37^{(-)}$ & $0,26^{(+)}$ & & \\
\hline Asociación Religiosa & & & & & & & \\
\hline
\end{tabular}

Correlación de Pearson

${ }^{*} p<0,05$.

${ }^{* *} p<0,01$.

Tabla 2.

Análisis de Regresión Lineal de los rasgos de personalidad y los valores sobre el grado de satisfacción con la vida y la pertenencia a alguna asociación religiosa

\begin{tabular}{|c|c|c|c|c|c|}
\hline \multirow{2}{*}{$\begin{array}{l}\text { Variable dependiente: } \\
\text { Variables predictoras }\end{array}$} & \multicolumn{5}{|c|}{ Grado de Satisfacción con la vida } \\
\hline & $R$ & $\mathrm{R}^{2}$ & $\mathrm{R}^{2}$ corregida & Error típ. de estimación & Valor $\mathrm{F}$ \\
\hline Rasgos de Personalidad &, 41 &, 17 &, 16 & 1,16 & $23,08^{* *}$ \\
\hline Valores Personales & 29 &, 09 & 08 & 1,22 & $10.59^{* *}$ \\
\hline Variable dependiente: & \multicolumn{5}{|c|}{ Pertenencia a Asociación Religiosa } \\
\hline Variables predictoras & $R$ & $\mathrm{R}^{2}$ & $\mathrm{R}^{2}$ corregida & Error típ. de estimación & \\
\hline Valores Personales &, 27 &, 07 & 07 &, 59 & $9,06^{* *}$ \\
\hline Rasgos de Personalidad &, 24 &, 06 &, 05 &, 60 & $6,66^{*}$ \\
\hline
\end{tabular}

* Nivel de significación $<0,01$.

** Nivel de significación $<0,001$.

el mayor porcentaje de varianza explicada correspondía a los valores personales frente a los rasgos de personalidad (7\% versus 5\%).

\section{DISCUSIÓN}

En el presente estudio se confirma la relación entre los valores personales y los rasgos de personalidad en pacientes con dependencia de la cocaína. Tal y como se estableció en la primera hipótesis, el rasgo energía se relaciona con los valores de la dimensión autopromoción. La relación de este rasgo con los valores poder y logro, indicaría que los individuos que tienden a la actividad valoran igualmente la consecución de metas personales, e incluso podrian desear cierto control sobre el medio. Sin embargo, este rasgo se relacionó, además, con algunos valores de las otras tres dimensiones del modelo de valores de Schwartz, siendo, de los rasgos de personalidad analizados, el que mostró relación con un mayor número de valores personales del conjunto total. Esto podría sugerir que la mayoría de los valores implican acción, o están expresados en estos términos.

La segunda hipótesis también se ve confirmada en los resultados; el rasgo apertura mental, que implica una actitud receptiva hacia diferentes valores y situaciones, se relaciona con los valores de las dimensiones apertura al cambio y autotrascendencia, lo cual estaría indicando que estas personas valoran el pensamiento independiente, la creatividad y la exploración y estarían más abiertas a nuevas culturas, aprendizajes y experiencias. 
Del mismo modo, se confirmó la tercera hipótesis, ya que el rasgo afabilidad se asoció con los valores de autotrascendencia. Así, las personas "afables" buscarían antes el bienestar para la colectividad que para ellos mismos, en lugar de valorar el poder y los logros personales. El rasgo afabilidad mostró, además, una asociación significativa con el valor autodirección, lo cual podría estar relacionado con la proximidad, en el modelo de Schwartz, de este valor con los valores de autotrascendencia.

Sin embargo, contrariamente a lo planteado en la cuarta hipótesis de la investigación, el rasgo tesón no se relacionó con los valores de autopromoción, sino que mostró una asociación significativa con valores de seguridad, autodirección, benevolencia y universalismo. Por esta razón, deberíamos considerar la posibilidad de que aquellas personas que destacan por comportamientos de orden, reflexividad y escrupulosidad, no valoran tanto el alcanzar logros y conseguir cierto control, como la estabilidad y la seguridad en todos sus aspectos (valor seguridad), el pensamiento independiente y la creatividad (valor autodirección), y el bienestar de las personas, tanto de su endogrupo (valor benevolencia), como del exogrupo (valor universalismo).

La quinta hipótesis de la investigación fue confirmada, mostrando la relación del rasgo estabilidad emocional con el valor seguridad, de la dimensión conservación, lo cual podría indicar la importancia que el sentirse protegido y dentro de un ambiente previsible posee para la estabilidad emocional de las personas en tratamiento por dependencia de la cocaína.

Por otra parte, respecto a la especificidad de valores y rasgos de personalidad, se comprueba que los valores personales explican mejor la religiosidad, mientras que los rasgos de personalidad parecen explicar mejor la satisfacción vital. Por lo tanto, se evidencia que a pesar de las importantes relaciones encontradas entre ambos constructos, los aspectos de la conducta humana que discriminan son distintos, por lo que al ser usados en conjunto, permitirian tener una perspectiva más amplia de las motivaciones personales. Así, tal y como han adelantado otros autores (Bilsky y Schwartz, 1994; Caprara, Schwartz, Capanna, Vecchione y Barbaranelli, 2006; Roccas et al, 2002), los valores personales podrian influir más directamente en actitudes y conductas que están bajo control cognitivo, mientras que los rasgos de personalidad podrian afectar más fuertemente a tendencias y conductas sujetas a un reducido control cognitivo. Esto implicaría que, aun poseyendo unas capacidades explicativas distintas, ambos conceptos resultarían ser complementarios y de especial interés para la comprensión del comportamiento social.

Por lo tanto, reconociendo que la dependencia de sustancias es un problema multifactorial (Becoña, 2002), los resultados aquí obtenidos sugieren que los rasgos de personalidad y los valores personales son dos variables que guardan significativas asociaciones entre sí en una muestra de personas dependientes de la cocaína, pero que a su vez, se comportan de manera diferente en la explicación de distintas conductas (religiosidad y satisfacción vital). Además, teniendo en cuenta que estas variables han demostrado su utilidad en estudios previos que las relacionaban con el consumo de sustancias (Galdós y Sánchez, 2010; Pedrero 2002; Pons, Berjano y García, 1996), y dado que el inicio en el consumo de cocaína parece estar asociado con un alto control cognitivo, mientras que el establecimiento de la adicción se relaciona con menor control cognitivo (Saiz, 2008), es probable que resulte conveniente el considerarlos conjuntamente, como factores complementarios, en el desarrollo de programas de investigación e intervención sobre el consumo de cocaína.

Finalmente, resulta necesario tener en cuenta que, aunque la importancia de utilizar los rasgos y valores conjuntamente ya ha sido subrayada por distintos autores (Furnham, 1984; Snyder y Cantor, 1998), la relación que guardan apenas está empezando a ser abordada (Bilsky y Schwartz, 1994; Olver y Mooradian, 2003; Roccas, Sagiv, Schwartz y Knafo, 2002), por lo que serian necesarias investigaciones que profundicen en la relación de estas variables en contextos clínicos.

\section{AGRADECIMIENTOS}

El primer autor debe expresar su gratitud a la Fundación Ramón Areces, dado que su contribución económica hizo posible el trabajo de investigación de campo que este estudio implica. También debemos agradecer la ayuda recibida por parte de la Agencia Antidroga de la Comunidad de Madrid y de los distintos centros de rehabilitación que participaron en la presente investigación.

\section{REFERENCIAS}

Becoña, E. (2002). Bases científicas de la prevención de las drogodependencias. Madrid: PNSD.

Berrocal, C., Ortiz-Tallo, M., Fierro, A. y Jiménez, J. A. (2001). Variables clínicas y de personalidad en adictos a heroína. Anuario de Psicología, 32, 67-87.

Bilsky, W., y Schwartz, S. (1994). Values and personality. European Journal of Personality, 8, 163-181.

Caprara, G. V., Barbaranelli, C., y Borgogni, L. (2001). Cuestionario "Big Five". Madrid: TEA.

Caprara, G., Schwartz, S., Capanna, C., Vecchione, M., y Barbaranelli, C. (2006). Personality and Politics: Values, Traits, and Political Choice. Political Psychology, 27, 2-28.

Caprara, G., Vecchione, M. y Schwartz, S. (2009). Mediational role of values in linking personality traits to political orientation. Asian Journal of Social Psychology, 12, 82-94.

Digman, J. M. (1990). Personality structure: emerge of the five-factor model. Annual Review of Psychology, 41, 417-440.

Digman, J., y Takemoto-Choch, N. (1981). Factors in the natural language of personality: re-analysis, comparison, and interpretation of six major studies. Multivariate Behavioral Research, 16, 149-170. 
Furnham, A. (1984). Personality and values. Personality and Individual Differences, 5, 548-485.

Galdós, J. S. (2009). Estudio empírico de las variables de la Teoría de la Conducta Planificada como factores de riesgo para el consumo de cocaina en tres grupos diferentes, Adicciones, 21, 187-194.

Galdós, J. S. y Sánchez, I. M. (2010). Relación del tratamiento por dependencia de la cocaina con los valores personales de apertura al cambio y conservación. Adicciones, 22, 51-58.

Garzón, A., y Garcés, J. (1989). Hacia una conceptualización del valor. En A. Rodríguez y J. Seoane. Creencias, Actitudes y Valores (pp. 365-407). Madrid: Alhambra Universidad.

Goldberg, L. R. (1990). An alternative "Description of Personality"; the Big-Five Factor structure. Journal of Personality and Social Psychology, 59, 1216-1229.

Gómez, A., Gaviria, E., y Fernández, I. (2006). Psicología Social. Madrid: Sanz y Torres.

Liu, H., Yu, S., Cottrell, L., Lunn, S., Deveaux, L., Brathwaite, N. et al. (2007). Personal values and involvement in problem behaviors among Bahamian early adolescents: a cross-sectional study. BMC Public Health, 7, 135.

Luciano, C., Páez-Blarrina, M. y Valdivia-Salas, S. (2010). La Terapia de Aceptación y Compromiso (ACT) en el consumo de sustancias como estrategia de Evitación Experiencial. Internacional Journal of Clinical and Health Psychology, 10, 141-165.

Luk, C. y Bond, M. (1993). Personality Variation and Values Endorsement in Chinese University Students. Personality and Individual Differences, 14, 429-437.

Martínez, I., y Garcia, J. F. (2007). Impact of parenting styles on adolescents' self-esteem and internalization of values in Spain. The Spanish Journal of Psychology, 10, 338-348.

Martínez, I., y Garcia, J. F. (2008). Internalization of values and self-esteem among Brazilian teenagers from authoritative, indulgent, authoritarian, and neglectful homes. Adolescence, 43, 13-29.

McCrae, R. (2001). Facts and interpretations of personal trait stability: a reply to Quackenbush. Theory and Psychology, 11, 837-844.

McCrae, R., y John, O. (1992). An Introduction to the Five-Factor Model and its Applications. Journal of Personality, 60, 175-215.

Morales, E., Ariza, C., Nebot, M., Pérez, A. y Sánchez, F. (2008). Consumo de cannabis en los estudiantes de secundaria de Barcelona: inicio en el consumo, efectos experimentados y expectativas. Gaceta Sanitaria, 22, 321-329.

Observatorio Español sobre Drogas. (2007). Informe 2007. Madrid: Ministerio del Interior.

Olver, J., y Mooradian, T. (2003). Personality traits and personal values: a conceptual and empirical integration. Personality and Individual Differences, 35, 109-125.

Pedrero, J. (2002). Evaluación de la personalidad de sujetos drogodependientes que solicitan tratamiento mediante el BigFive Questionnaire. Trastornos Adictivos, 4, 138-150.

Pedrero, J. (2003). Los trastornos de la personalidad en drogodependientes desde la perspectiva de los cinco grandes factores. Adicciones, 15, 203-220.
Pérez, E. J. P.; Mota, G. R. y Arroyo, A. O. (2008). Valores sociales y adicción: aplicabilidad y propiedades psicométricas del cuestionario VAL-89. Adicciones, 20, 327-336.

Pons, J. y Berjano, E. (1999). El consumo abusivo de alcohol en la adolescencia: un modelo explicativo desde la Psicología Social. Madrid: PNSD.

Pons, J., Berjano, E. y García, F. (1996). Variables psicosociales que discriminan el consumo abusivo de alcohol en la adolescencia. Adicciones, 8, 177-191.

Roccas, S., Sagiv, L., Schwartz, S., y Knafo, A. (2002). The Big Five Personality Factors and Personal Values. Personality and Social Psychology Bulletin, 28, 789-801.

Ros, M. (2001). Psicología Social de los Valores: una perspectiva histórica. En M. Ros y V. V. Gouveia (Coords.). Psicología social de los valores humanos (pp. 27-51). Madrid: Biblioteca Nueva.

Saiz, J. (2007). El abuso de cocaina, ¿Problema de oferta o de demanda social?: un estudio transcultural y correlacional que compara variables macrosociales, económicas y culturales. Adicciones, 19, 35-44.

Saiz, J. (2008). Un estudio del consumo de cocaína en la Comunidad de Madrid desde la Psicología Social. Madrid: UCM, Servicio de Publicaciones.

Schwartz, S. (2001). ¿Existen aspectos universales en la estructura y contenido de los valores humanos? En M. Ros y V. V. Gouveia (Coords.). Psicología social de los valores humanos (pp. 53-77). Madrid: Biblioteca Nueva.

Schwartz, S., y Bilsky, W. (1987). Toward a Universal Psychological Structure of Human Values. Journal of Personality and Social Psychology, 53, 550-562.

Schwartz, S., Melech, G., Lehmann, A., Burgess, S., Harris, M., y Owens, V. (2001). Extending the cross-cultural validity of the theory of basic values with a different method of measurement. Journal of Cross-Cultural Psychology, 32, 519-542.

Schwartz, S., Verkasalo, M., Antonovsky, A., y Sagiv, L. (1997). Values priorities and social desirability: much substance, some style. British Journal of Social Psychology, 36, 3-18.

Snyder, M., y Cantor, N. (1998). Understanding personality and social behavior: a functionalist strategy. En D. T. Gilbert, S. T. Fiske y G. Lindzey. The Handbook of Social Psychology (pp. 635-685). USA.: McGraw-Hill.

Spini, D. (2003). Measurement equivalence of 10 value types from the Schwartz value survey across 21 countries. Journal of CrossCultural Psychology, 34, 3-23.

Terracciano, A., Löckenhoff, C., Crum, R., Bienvenu, J. y Costa, P. (2008). Five-Factor Model personality profiles of drug users. BMC Psychiatry, 8, 22.

Tupes, E., y Christal, R. (1961/1992). Recurrent Personality Factors Based on Trait Ratings. Journal of Personality, 60, 225-251.

Zlobina, A. (2004). Estereotipos nacionales y regionales en Europa y España. En D. Páez, I. Fernández, S. Ubillos y E. Zubieta (Eds.). Psicología social, cultura y educación. Madrid: Prentice Hall. 